\title{
Revisão de literatura: odontologia preventiva em pacientes ortodônticos - como prevenir e tratar as lesões de mancha branca?
}

Preventive dentistry in orthodontic patients - how to prevent and treat white spot lesions? a review

RODRIGUES, Lívia Pagotto'; FREITAS, Fabiana Furtado ; ZANCOPÉ, Bruna Raquel'; CALDARELLI, Pablo Guilherme'; PEREIRA, Antônio Carlos ${ }^{2}$; BULGARELI, Jaqueline Vilela ${ }^{1}$

1. Doutorando em Odontologia, Faculdade de Odontologia de Piracicaba, Universidade Estadual de Campinas, Piracicaba, SP, Brasil.

2. Professor Titular da área de Odontologia em Saúde Coletiva, Faculdade de Odontologia de Piracicaba, Universidade Estadual de Campinas, Piracicaba, SP, Brasil

Endereço para correspondência:

Jaqueline Vilela Bulgareli

Av. Limeira, 901

13414-903 - Piracicaba - São Paulo - Brasil

Email: jaquelinebulgareli@gmail.com

Recebido: 15.12 .2015

Aceito: 30.06.2017

\section{RESUMO}

Os problemas de maloclusão são considerados como o terceiro maior problema de saúde pública pela Organização Mundial de Saúde e a busca pela correção de desvios morfológicos da oclusão normal tem feito com que a procura por tratamento ortodôntico seja cada vez maior. Entretanto, um dos maiores problemas relacionados ao uso de dispositivos ortodônticos tem sido o desenvolvimento de lesões de mancha branca, que é considerada como a precursora da lesão de cárie cavitada e deve ser corretamente diagnosticada e prevenida. Assim, o presente estudo tem como objetivo realizar uma revisão narrativa da literatura sobre a etiologia, o tratamento e a prevenção das lesões de mancha branca em pacientes ortodônticos. Trata-se de um estudo descritivo e exploratório, realizado por meio de um levantamento em bases eletrônicas de domínio público. Concluiu-se que a colaboração do paciente e o uso de bochecho com solução de fluoreto de sódio a $0.05 \%$ torna-se uma associação terapêutica de sucesso, permitindo que o resultado final do tratamento seja satisfatório em termos funcionais, estéticos e de promoção da saúde bucal.

Palavras-chave: Odontologia preventiva. Ortodontia. Desmineralização.

\section{ABSTRACT}

Malocclusion problems are considered as the third bigger public health problem by the World Health Organization and the search for correction of morphological deviations from normal occlusion has caused the increasing on demand for orthodontic treatment. However, a major problem related to the use of orthodontic appliances has been the development of white spot lesions. It is considered as the precursor of the cavitated caries lesion and must be correctly diagnosed and prevented. Thus, the aim of this study is to conduct a narrative review of the literature about the etiology, treatment and prevention of white spot lesions in orthodontic patients. It is a descriptive and exploratory study, conducted through a survey in electronic databases in the public domain. It was concluded that the cooperation of the patient and the use of mouthwash with sodium fluoride $0.05 \%$ solution becomes a combination therapy of success, allowing the final outcome be satisfactory in terms of functional, aesthetic and promotion oral health.

Keywords: Preventive dentistry. Orthodontics. Demineralization. 


\section{INTRODUÇÃO}

Com a popularização da Ortodontia nas últimas três décadas, muitos pacientes estão sendo beneficiados com o tratamento ortodôntico' ${ }^{\prime}$. A razão principal que motiva a maioria dos pacientes a procurarem por esse tratamento é a busca por estética, seguida pelas indicações médicas e odontológicas $^{2}$. Entretanto, um dos afeitos adversos do uso de aparelhos ortodônticos fixos é o acúmulo de biofilme dental ao redor dos dispositivos colados ao dente, que está associado com o risco aumentado de desenvolvimento de lesões de manchas brancas ${ }^{3}$. Este achado clínico é apontado como um dos riscos do tratamento ortodôntico ${ }^{4.7}$. Neste contexto, as lesões de manchas brancas associadas ao uso do aparelho ortodôntico fixo têm sido relatadas como um dos maiores problemas de relevância clínica ${ }^{4,8-13}$, as quais acometem aproximadamente $50 \%$ dos pacientes em tratamento ortodôntico ${ }^{3}$. Este impacto clínico se deve ao fato de que a lesão de mancha branca é considerada como a precursora da lesão de cárie cavitada ${ }^{14-15}$, podendo também ser considerada um problema estético relevante.

Além disso, as lesões de manchas brancas são uma complicação comum decorrente de higiene oral deficiente pela dificuldade de limpeza das superfícies dentais em virtude da presença dos dispositivos ortodônticos e gera percepções negativas quanto ao resultado final do tratamento ${ }^{16}$ tendo em vista que a terapia ortodôntica tem como finalidade não apenas a oclusão funcional, mas também harmonia, estética e saúde da cavidade bucal. Assim, o sucesso do tratamento ortodôntico é relacionado à correção da oclusão de maneira satisfatória, sem, contudo, alterar a higidez pré-existente dos dentes e tecidos de suporte, por meio da aplicação de um modelo de programa educativo-preventivo individualizado a cada paciente, que considere os fatores de risco à cárie dentária, doença periodontal e seu potencial de colaboração com as medidas empregadas $^{17}$. Prevenir a formação das lesões de mancha branca decorrentes do tratamento ortodôntico deve ser uma preocupação constante do ortodontista.

Assim, o objetivo deste trabalho é realizar uma revisão da literatura acerca da etiologia da lesão de mancha branca, bem como de estratégias preventivas, métodos de tratamento dessas lesões ao redor de dispositivos ortodônticos e seus impactos no resultado final do tratamento.

Trata-se de um estudo descritivo e exploratório, no qual a estratégia de identificação e seleção dos estudos foi levantamento bibliográfico de publicações indexadas em bases eletrônicas de dados: Lilacs (Literatura LatinoAmericana e do Caribe em Ciências da Saúde), MEDLINE (Medical Literature and Retrivial System on Line) e SciELO (Scientific Electronic Library Online). Foram adotados os seguintes critérios para seleção dos artigos: todas as categorias de artigo (original, revisão de literatura, reflexão, atualização e relatos de casos clínicos), artigos com resumos e textos completos disponíveis para análise, publicados nos idiomas português, inglês e espanhol que abordassem a temática. Não foi realizada a inclusão do filtro relacionado à data das publicações, uma vez que existem estudos pioneiros na área, que são considerados um marco para as pesquisas seguintes e que foram incluídos, além dos artigos encontrados pelas ferramentas de busca citados.

Foram selecionados os artigos que continham em seus títulos e/ou resumos os seguintes descritores em ciências da saúde (DeCS): aparelho ortodôntico, lesão de mancha branca e cárie. Após, procedeu-se à leitura minuciosa de cada artigo científico, destacando aqueles que responderam aos objetivos propostos por este estudo. Para isso, foi elaborado um instrumento de coleta de dados contendo título do artigo científico, título do periódico, ano de publicação, país do estudo, categoria do estudo, natureza do estudo, referencial teórico, método de análise, objetivo e resultados encontrados. Ao todo, foram encontrados 18 artigos, seguindo os critérios previamente estabelecidos. Foram excluídos artigos cuja metodologia foi considerada distante da proposta deste estudo.

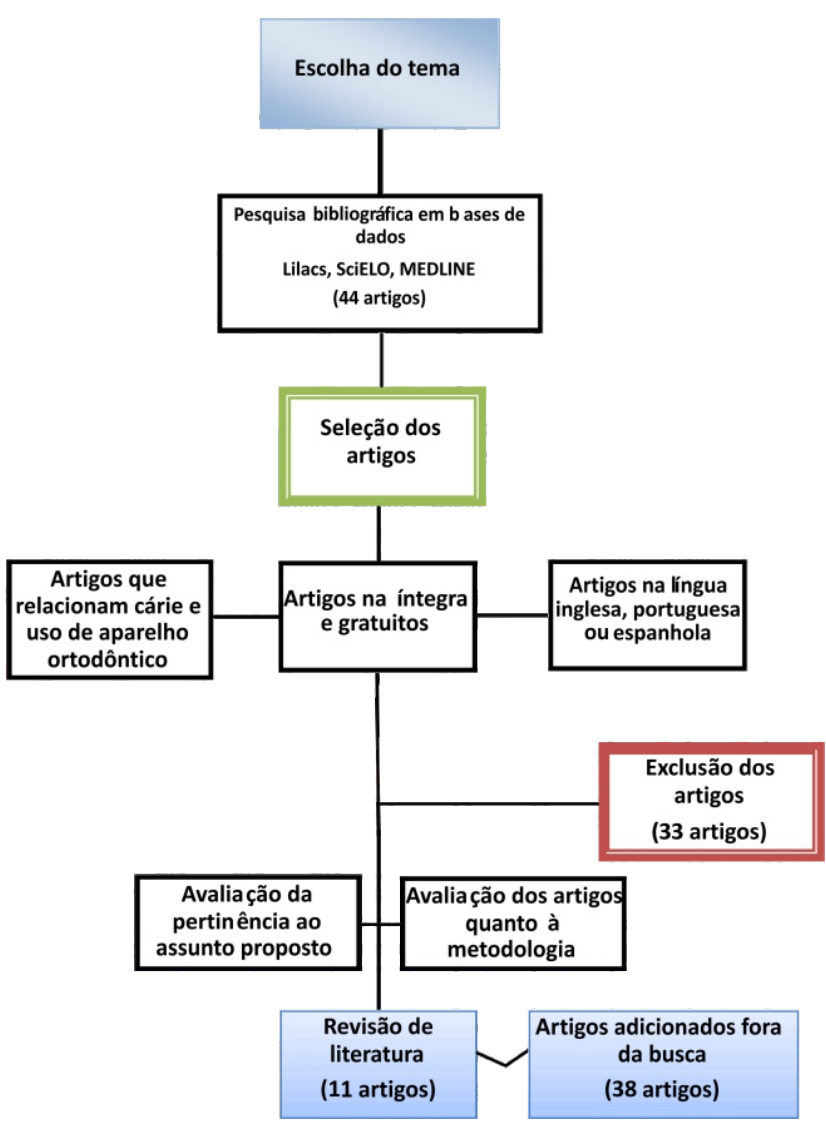

Figura 1 - Diagrama ilustrando a metodologia utilizada na busca bibliográfica. 


\section{REVISÃO DE LITERATURA}

\section{Etiologia das Lesões de Manchas Brancas por Desmineralização}

Um dos maiores problemas relacionados ao uso de dispositivos ortodônticos tem sido o desenvolvimento de lesões de mancha branca, que é considerada como a precursora da lesão de cárie cavitada e deve ser corretamente diagnosticada e prevenida ${ }^{14-15}$. Sabe-se que o desenvolvimento de uma lesão de cárie é um processo dinâmico, sendo caracterizado por períodos alternados de desmineralização e remineralização, que ocorrem constantemente entre os tecidos dentários e os fluidos do meio bucal ${ }^{18-19}$. Constantemente há trocas minerais, e estas são influenciadas pelas condições que mantêm um pH crítico na cavidade bucal. Ou seja, desde que seja mantido na cavidade bucal pH maior que 5.5, haverá condição de supersaturação da saliva, que contém cálcio $(\mathrm{Ca})$ e fosfato (P), superando o produto de solubilidade da hidroxiapatita (HA). Assim, a tendência físico-química é o ganho de minerais pelo tecido dental, oriundos do meio-bucal. Conceitua-se o $\mathrm{pH}$ de 5,5 como $\mathrm{pH}$ crítico, pois este é o limite do produto iônico das concentrações de $\mathrm{Ca}$ e $\mathrm{P}$ na saliva, que favorece o equilíbrio entre o processo de desremineralização ${ }^{18}$.

Quando o pH na cavidade bucal é menor que 5.5, a composição da saliva em relação ao Ca e P torna-se inferior (subsaturante) em relação ao produto de solubilidade da HA, e, deste modo, a tendência físico-química é a perda de íons Ca e P do esmalte para o meio bucal, na tentativa de atingir o novo estado de equilíbrio em função do $\mathrm{pH}$ atingido. A queda do $\mathrm{pH}$ no biofilme dental ocorre após ingestão de carboidratos solúveis (sacarose), ocorrendo como consequência a dissolução do esmalte ${ }^{20}$.

A lesão de cárie é o sinal clínico do desequilíbrio do processo desremineralização, quando há predominância da perda mineral do esmalte e da dentina, sendo definida como resultado da dissolução química dos tecidos duros do dente, provocada por ácidos provenientes do consumo de açúcar com baixo peso molecular. Pode ser classificada desde o início da perda mineral, podendo se estender até a destruição total do dente ${ }^{21}$. Quando em estágio inicial, a lesão de cárie pode ser totalmente remineralizada e isto enfatiza a necessidade do aperfeiçoamento de métodos preventivos que possam agir como coadjuvantes na prevenção e controle da cárie dentária em pacientes ortodônticos ${ }^{22}$.

Os dispositivos criam novas áreas de retenção para a formação de biofilme, cujo acúmulo, na presença de açúcares da dieta poderia resultar em um aumento do número e porcentagem de espécies cariogênicas, como Streptococcus mutans e Lactobacillus ${ }^{4,15}$. O aumento da população de bactérias cariogênicas pode levar a uma queda no $\mathrm{pH}$, que altera o balanço do equilíbrio dos processos de desmineralização-remineralização, levando assim, à perda de minerais, que pode acarretar no desenvolvimento de manchas brancas e, eventualmente, cavitação, podendo se estender até a dentina. Em casos mais severos pode haver a necessidade de tratamento restaurador destas lesões, quando cavitadas. A porcentagem de pacientes que desenvolvem lesões de mancha branca decorrentes do tratamento ortodôntico é citada em trabalhos na literatura como variando em torno de $50 \% 0^{4,23-26}$.

As lesões de mancha branca comprometem a estética dentária, podendo ocorrer por cárie incipiente, opacidades do esmalte ou hipoplasia do esmalte. Nessas situações, há uma diminuição do conteúdo mineral e modificação da organização estrutural, havendo assim um aumento do conteúdo orgânico do esmalte, o que promove a redução da resistência física do tecido ${ }^{11}$. A aparência é causada por um fenômeno óptico devido à perda de minerais e substituição do conteúdo orgânico da subsuperfície por ar, assim, quando da secagem exagerada, evidencia-se a mancha ${ }^{4}$.

Realizou-se um estudo com o objetivo de avaliar a etiologia e prevenção da desmineralização do esmalte durante o tratamento ortodôntico ${ }^{27}$. Estes autores citam fatores etiológicos gerais da cárie dentária, como: os fatores microbianos, fatores salivares, o fluxo salivar, o $\mathrm{pH}$ e a capacidade tampão da saliva, a higiene bucal e a dieta. Dentre os fatores etiológicos mais específicos em relação ao tratamento ortodôntico, os a própria influência da presença do dispositivo ortodôntico fixo gera uma área de estagnação, favorecendo o acúmulo de biofilme e servindo como uma fonte de produção de ácidos na presença de substratos alimentares ${ }^{27-28}$.

\section{Meios Preventivos}

A literatura é vasta a respeito dos métodos preventivos ao desenvolvimento de áreas de desmineralização ao redor de dispositivos ortodônticos, podendo ser citados a educação em saúde bucal do paciente ortodôntico, administração de agentes fluoretados e uso de materiais de colagem liberadores de fluoreto, durante o tratamento ${ }^{17,29-36}$.

\section{Agentes Fluoretados}

Vários autores sugeriram métodos preventivos para prevenir a desmineralização ao redor dos dispositivos ortodônticos, sendo citados a constante instrução de higiene bucal e o uso de bochechos fluoretados. O uso de fluoretos é um recurso importante na prevenção da desmineralização do esmalte e atualmente, há várias formas de uso deste composto durante o tratamento ortodôntico, incluindo o uso de dentifrícios, bochechos, géis, vernizes e materiais liberadores de flúor, tais como adesivos e elásticos ${ }^{29,37-38}$.

Em um estudo clínico, objetivaram quantificar e 
avaliar a extensão da desmineralização ao redor de dispositivos ortodônticos após um mês de uso do aparelho na boca e também, avaliar a habilidade de produtos à base de fluoretos disponíveis no mercado de inibir ou reverter o processo de desmineralização. $\mathrm{O}$ estudo mostrou forte evidência da efetividade da combinação do uso de dentifrício fluoretado diariamente associado com o uso de bochecho fluoretado $(0.05 \%$ de fluoreto de sódio) contra o processo de desmineralização e promoção da remineralização quando do emprego contínuo desses dois métodos de uso do fluoreto ${ }^{15}$.

Em um estudo clínico verificou-se a frequência de bochechos com fluoreto de sódio $0.05 \%$ influenciava na formação de lesões de mancha branca no esmalte de pacientes que utilizavam bráquetes ortodônticos. Concluiu-se que redução significante de lesões de manchas brancas no esmalte pode ser alcançada durante o tratamento ortodôntico através do uso de $10 \mathrm{~mL}$ de solução para bochecho à base de fluoreto de sódio a $0.05 \% 39$.

O flúor não deve ser considerado apenas como uma substância preventiva contra a doença cárie, mas também, uma substância terapêutica que pode ser um agente auxiliar no processo de remineralização das lesões. $\mathrm{O}$ autor sugere ainda, que o ortodontista não deve confiar apenas no uso de bochechos fluoretados, embora estudos controlados comprovem que a incidência de manchas brancas pode ser reduzida com os mesmos. Assim, conclui que seria melhor utilizar vernizes de flúor que podem ser aplicados nas superfícies que apresentam maior risco à desmineralização, três ou mais vezes por ano, dependendo do grau de risco à doença. Adicionalmente, o trabalho sugere que deve ser aplicado verniz fluoretado nas superfícies dentais, tanto antes da colocação das bandas ortodônticas, quanto durante o tratamento e na remoção dos acessórios ${ }^{40}$.

Alguns estudos têm concluído que apenas o dentifrício fluoretado não tem sido suficiente para evitar o aparecimento das lesões de mancha branca em pacientes ortodônticos e que seria necessária a suplementação com o uso de bochechos diários e/ou uso de selantes em superfícies suscetíveis ao desenvolvimento de lesões de mancha branca, antes da colagem dos bráquetes ${ }^{27}$.

Atualmente há um consenso de que o flúor importante é aquele mantido constante na cavidade bucal, o qual é capaz de interferir na dinâmica do processo de cárie, reduzindo a quantidade de minerais perdidos durante o processo de desmineralização e ativando a quantidade de resposta durante a remineralização salivar ${ }^{18}$.

Em uma revisão sistemática de literatura avaliou-se a efetividade dos fluoretos na prevenção da ocorrência de lesões de mancha branca em pacientes ortodônticos. Os autores avaliaram estudos que utilizaram diversas formas de uso de fluoretos e encontraram evidência de que o uso diário de bochecho fluoretado é capaz de reduzir a severidade da desmineralização. Baseados nos estudos avaliados, os autores sugerem que a melhor opção de recomendação de uso de fluoretos como método preventivo seria o uso de bochechos diários contendo fluoreto de sódio a $0.05 \%{ }^{29}$.

\section{Materiais de Colagem Liberadores de Fluoreto}

O procedimento de colagem em esmalte dental baseia-se em alterações morfológicas ocorridas no esmalte em decorrência da ação de tratamentos superficiais propostos inicialmente por Buonocore ${ }^{41}$. O autor verificou que a ação de substâncias ácidas sobre a estrutura mineral possibilita a retenção micromecânica de materiais resinosos ao esmalte dental previamente condicionado. Tal descoberta também foi empregada na Ortodontia, promovendo grandes benefícios clínicos, uma vez que este mecanismo de união permanece sendo usado quando na colagem de acessórios ortodônticos.

O comportamento clínico da cimentação de bráquetes com ionômero de vidro (SHOFU 1) foi analisado por um período de até 18 meses. Para isso, os acessórios ortodônticos foram colados em 80 dentes de 10 pacientes com idades compreendidas entre 14 e 17 anos. A manipulação do material de colagem foi realizada seguindo-se as recomendações do fabricante. Após quatro meses do início do estudo, os autores observaram que dez bráquetes haviam se soltado, sendo necessário, portanto, nova cimentação do acessório, com o mesmo material previamente utilizado. Oito acessórios se soltaram após 13 meses de avaliação e, após 17 meses, apenas um bráquete. Os autores concluíram que o cimento de ionômero de vidro SHOFU I mostrou-se eficiente na colagem de bráquetes ortodônticos. O material empregado no estudo mostrou-se capaz de prevenir o desenvolvimento de desmineralização do esmalte ao redor de bráquetes ortodônticos, comumente observada ao fim do tratamento ortodôntico ${ }^{42}$.

Avaliou-se in vivo o potencial cariostático de um adesivo fotoativável utilizado para a colagem de bráquetes ortodônticos. A liberação de flúor do material foi avaliada tanto em água quanto em saliva. Dez voluntários possuindo pré-molares indicados para a extração participaram do estudo. Foram avaliados os materiais Heliosit-Orthodontic (Vivadent, Liechtenstein), não liberador de flúor, e o cimento ortodôntico VP 862 (Vivadent, Liechtenstein), que apresenta uma liberação de flúor lenta. Os voluntários foram instruídos a utilizar dentifrício fluoretado durante o experimento. Após quatro semanas de estudo, os dentes previamente selecionados foram extraídos e o conteúdo mineral do esmalte ao redor dos bráquetes foi avaliado por microrradiografia. A liberação de flúor de discos confeccionados em laboratório com o material adesivo liberador de flúor foi avaliada em água, após um período de seis meses e em saliva, após um período de 24 horas. $\mathrm{O}$ material liberador de flúor reduziu a profundidade da lesão em $48 \%$ em relação ao adesivo não liberador de flúor. A maior liberação de flúor do material em água foi observada 
na primeira semana, entretanto, uma quantidade significante de flúor ainda era liberada mesmo após os seis meses de experimento. A liberação de flúor foi significativamente menor em saliva humana em um $\mathrm{pH}$ sete do que em água, entretanto, quando o $\mathrm{pH}$ salivar atingia um valor inferior a quatro, mimetizando um desafio cariogênico, essa quantidade de flúor aumentava, se assemelhando aos valores observados em água. Como conclusão do estudo, os autores afirmam que a liberação de flúor é $\mathrm{pH}$ dependente e ainda, que o uso regular de dentifrício fluoretado é insuficiente para inibir o desenvolvimento de lesões de mancha branca ao redor de bráquetes ortodônticos ${ }^{43}$.

Em um estudo in vivo, realizaram uma análise quantitativa dos efeitos do cimento de ionômero de vidro sobre os dentes com bráquetes ortodônticos, que tinham indicação de extração por razões ortodônticas. Para isso, eles avaliaram a porcentagem de perda mineral, que foi calculada baseada no quanto de desmineralização houve e ainda, avaliaram as concentrações de flúor de amostras de saliva coletadas dos pacientes. Os voluntários foram instruídos a escovar seus dentes duas vezes ao dia com um dentifrício fluoretado (1100 ppm F). Como material não liberador de flúor, os autores utilizaram a resina Transbond XT (3M Unitek, Monrovia). Ao final de quatro semanas de experimento, os dentes previamente selecionados foram extraídos e armazenados em solução de timol a $0.1 \%$. Posteriormente, foi realizada a análise de microdureza longitudinal, seguindo o padrão em V. Os resultados de microdureza mostraram que houve significativamente maior desmineralização ao redor dos bráquetes do grupo que utilizou o material de colagem não liberador de flúor. Em relação à analise salivar, oito amostras foram coletadas de cada paciente ao longo das quatro semanas, não havendo diferença significativa entre os grupos testados quanto à concentração de flúor na saliva ${ }^{9}$.

Avaliou-se in vivo o efeito do cimento de ionômero de vidro na redução da desmineralização ao redor de bráquetes ortodônticos. Para o estudo, 14 voluntários foram selecionados e randomicamente divididos em 2 grupos, sendo que 7 tiveram bráquetes colados aos pré-molares com a resina Concise (3M Produtos, St Paul, Minn) sendo este o grupo controle, e outros 7 voluntários tiveram os bráquetes colados com Fuji Ortho LC (GC American, Chicago III), um cimento de ionômero de vidro modificado por resina, sendo este o grupo experimental. Após 30 dias de experimento, os voluntários tiveram os pré-molares extraídos. Os dentes foram seccionados e avaliados por análise de microdureza longitudinal. Os autores concluíram ao final do estudo que o uso do cimento de ionômero de vidro para colagem de bráquetes foi estatisticamente mais significante em relação à diminuição da desmineralização ao redor dos dispositivos ortodônticos. Assim, os autores encorajam o uso do cimento de ionômero de vidro como material de colagem ${ }^{44}$.
O emprego de cimentos de ionômero de vidro para colagem de dispositivos ortodônticos reduz a ocorrência de lesões de mancha branca, quando comparados às resinas compostas ${ }^{29}$.

Uma revisão de literatura sistemática avaliou a efetividade de materiais adesivos contendo flúor no controle da desmineralização do esmalte dentário em pacientes em tratamento ortodôntico. Os autores encontraram evidência de que o uso do cimento de ionômero de vidro como material adesivo é mais efetivo em prevenir a desmineralização durante o tratamento ortodôntico, em relação às resinas compostas convencionais. Entretanto, devido às limitações de adesão do ionômero, o mesmo não pode ser recomendado. Ainda, os autores concluíram que mais estudos são necessários para avaliar a efetividade dos materiais adesivos que contêm flúor. Assim, os autores concluíram que o cimento de ionômero de vidro foi capaz de reduzir significativamente a desmineralização ao redor dos bráquetes ortodônticos, no período de quatro semanas de avaliação ${ }^{37}$.

\section{DISCUSSÃO}

Ainda que existam avanços em relação aos materiais e técnicas disponíveis na Ortodontia, a prevalência das lesões de manchas brancas em pacientes ortodônticos continua elevada, em torno de $50 \%{ }^{4,23-26}$. Assim, prevenir a ocorrência dessas lesões é um assunto de extrema importância.

A literatura é unânime em relação às terapias com flúor que combinam dentifrícios e bochechos fluoretados e aplicação profissional de flúor. Essas têm se mostrado eficazes em prevenir ou reduzir as lesões de manchas brancas em pacientes com aparelho ortodôntico ${ }^{29-30,34}$. No entanto, esse é um tratamento que apresenta baixo índice de cooperação por parte dos pacientes, o que compromete sua efetividade ${ }^{45-46}$, e o efeito do flúor é parcial já que o mesmo não consegue impedir completamente o desenvolvimento das lesões de cárie ${ }^{45}$. Adicionalmente, as recomendações aos pacientes devem incluir instrução de higiene bucal, reforço e controle da mesma, sempre que possível ${ }^{47}$.

Clinicamente, as lesões de manchas brancas com profundidade de 75 ?m podem ser desenvolvidas no período de apenas quatro semanas, o que implica dizer que a aplicação tópica de flúor deve ser mantida durante todo o tratamento, porém, normalmente isso não ocorre na clínica ${ }^{5}$. Assim, os fabricantes incorporaram flúor aos materiais de colagem ortodôntica com o objetivo de ajudar a prevenir a desmineralização do esmalte dental ao redor dos bráquetes ${ }^{23,48}$.

Materiais adesivos que apresentam liberação lenta de flúor promovem redução e até mesmo inibição do processo de desmineralização do esmalte adjacente ao material ${ }^{49-50}$. A vantagem principal de materiais com liberação lenta de flúor é relacionada à liberação inicial do fluoreto adjacente ao bráquete ortodôntico, sendo essa a área de maior risco à desmineralização do esmalte. 


\section{CONCLUSÃO}

Tomando-se as devidas precauções, é possível alcançar uma oclusão funcional e esteticamente aceitável sem deixar que a doença cárie tenha um papel coadjuvante no transcorrer e após o término do tratamento ortodôntico. Tais medidas encontram-se diretamente relacionadas à colaboração constante do paciente, uma vez que o bochecho com solução de fluoreto de sódio a $0.05 \%$ deve ser mantido durante todo o tratamento ortodôntico, bem como boas práticas de higiene bucal.

\section{REFERÊNCIAS}

1. Consolaro A, Consolaro MFM. Lesões cariosas incipientes e formação de cavidades durante o tratamento ortodôntico. Rev Clin Ortod Dent Press. 2006;5(4):104-11.

2. Ackerman MB. Selling orthodontic need: innocent business decision or guilty pleasure? J Med Ethics. 2010;36(5):275-8.

3. Tufekci E, Dixon JS, Gunsolley JC, Lindauer SJ. Prevalence of white spot lesions during orthodontic treatment with fixed appliances. Angle Orthod. 2011;81(2):206-10.

4. Gorelick L, Geiger AM, Gwinnett AJ. Incidence of white spot formation after bonding and banding. Am J Orthod. 1982;81(2):93-8.

5. Øgaard B. Prevalence of white spot lesions in 19-year-olds: a study on untreated and orthodontically treat persons 5 years after treatment. Am J Orthod Dentofacial Orthop. 1989;96(5):423-7.

6. Van der Kaaij NC, van der Veen MH, van der Kaaij MA, ten Cate JM. A prospective, randomized placebo-controlled clinical trial on the effects of a fluoride rinse on white spot lesion development and bleeding in orthodontic patients. Eur J Oral Sci. 2015;123(3):186-93.

7. Van der Linden RP, Dermaut LR. White spot formation under orthodontic bands cemented with glass ionomer with or without Fluor Protector. Eur J Orthod. 1998;20(3):219-24.

8. Al Maaitah EF, Adeyemi AA, Higham SM, Pender N, Harrison JE. Factors affecting demineralization during orthodontic treatment: a post-hoc analysis of RCT recruits. Am J Orthod Dentofacial Orthop. 2011;139(2):181-91.

9. Gorton J, Featherstone JD. In vivo inhibition of demineralization around orthodontic brackets. Am J Orthod Dentofacial Orthop. 2003;123(1):10-4.

10. Kukleva MP, Shetkova DG, Beev VH. Comparative age study of the risk of demineralization during orthodontic treatment with brackets. Folia Med (Plovdiv). 2002;44(1-2):56-9.

11. Lovrov S, Hertrich K, Hirschfelder U. Enamel demineralization during fixed orthodontic treatment - Incidence and correlation to various oralhygiene parameters. J Orofac Orthop. 2007;68(5):353-63.

12. Richter AE, Arruda AO, Peters MC, Sohn W. Incidence of caries lesions among patients treated with comprehensive orthodontics. Am J Orthod Dentofacial Orthop. 2011;139(5):657-64.
13. Zimmer B, Rottwinkel Y. Assessing patient-specific decalcification risk in fixed orthodontic treatment and its impact on prophylactic procedures. Am J Orthod Dentofacial Orthop. 2004;126(3):318-24.

14. Darling Al. Studies of the early lesion of enamel caries with transmitted light, polarized light and radiography. Parts I and II. Br Dent J 1956;101:289-97,324-41.

15. O'Reilly MM, Featherstone JDB. Demineralization and remineralization around orthodontic appliances: an in vivo study. Am J Orthod Dentofacial Orthop. 1987;92(1):33-40.

16. Maxfield BJ, Hamdan AM, Tüfekçi E, Shroff B, Best AM, Lindauer SJ. Development of white spot lesions during orthodontic treatment: perceptions of patients, parents, orthodontists, and general dentists. Am J Orthod Dentofacial Orthop. 2012;141(3):337-44.

17. Olympio KPK, Bardal PAP, Henriques JFC, Bastos JRM. Prevention of dental caries and periodontal disease in Orthodontics: a indispensable necessity. Rev Dent Press Ortod Ortop Facial. 2006;11(2):110-9.

18. Cury JA. Uso do flúor e controle da cárie como doença. In: Baratieri LN. Odontologia restauradora: fundamentos e possibilidades. São Paulo: Santos; 2001.p. 31-68.

19. Featherstone JD. The continuum of dental caries - evidence for a dynamic disease process. J Dent Res. 2004;83:39-42.

20. Fejerskov O, Nyvad B, Kidd EAM. Clinical and histological manifestation of dental caries. Oxford: Blackwell Munksgaard; 2003.p. 80-6.

21. Pitts NB. Modern concepts of caries measurement. J Dent Res. 2004;83:43-7.

22. Lussi A, Imwinkelried S, Pitts N, Longbottom C, Reich E. Performance of a laser fluorescence system for detection of occlusal caries in vitro. Caries Res. 1999;33(4):261-6.

23. Banks PA, Burn A, O'Brien K. A clinical evaluation of the effectiveness of including fluoride into an orthodontic bonding adhesive. Eur J Orthod. 1997;19(4):391-5.

24. Basdra EK, Huber H, Komposch G. Fluoride released from orthodontic bonding agents alters the enamel surface and inhibits enamel demineralization in vitro. Am J Orthod Dentofacial Orthop. 1996;109(5):466-72.

25. Boersma JG, van der Veen MH, Lagerweij MD, Bokhout B, PrahlAndersen B. Caries prevalence measured with QLF after treatment with fixed orthodontic appliances: influencing factors. Caries Res. 2005;39(1):41-7.

26. Øgaard B, Alm AA, Larsson E, Adolfsson U. A prospective, randomized clinical study on the effects of an amine fluoride/stannous fluoride toothpaste/mouthrinse on plaque, gingivitis and initial caries lesion development in orthodontic patients. Eur J Orthod. 2006;28(1):8-12

27. Chang HS, Walsh LJ, Freer TJ. Enamel demineralization during orthodontic treatment. Aetiology and prevention. Aust Dent J. 1997;42(5):322-7.

28. Martín IE, Corrales MC, Verdeja VE, Zaldívar LM. Factores de riesgo a caries en pacientes con aparatos ortodónticos fijos. AMC. 2010;14(5):1-11.

29. Benson PE,Shah AA, Millett DT, Dyer F, Parkin N, Vine RS. Fluorides, orthodontics and demineralization: a systematic review. J 
Orthod. 2005;32(2):102-12.

30. Cohen WJ, Wiltshire WA, Dawes C, Lavelle CL. Long-term in vitro fluoride release and rerelease from orthodontic bonding materials containing fluoride. Am J Orthod Dentofacial Orthop. 2003;124(5):571-6.

31. Korbmacher H, Huck L, Adam T, Kahl-Nieke B. Evaluation of an antimicrobial and fluoride-releasing self-etching primer on the shear bond strength of orthodontic brackets. Eur J Orthod. 2006;28(5):457-61.

32. Marcusson A, Norevall LI, Persson M. White spot reduction when using glass ionomer cement for bonding in orthodontics: a longitudinal and comparative study. Eur J Orthod. 1997;19(3):233-42.

33. Miwa H, Miyazawa K, Goto S, Kondo T, Hasegawa A. A resin veneer for enamel protection during orthodontic treatment. Eur J Orthod. 2001;23(6):759-67.

34. Nalbantgil D, Oztoprak MO, Cakan DG, Bozkurt K, Arum T. Prevention of demineralization around orthodontic brackets using two different fluoride varnishes. Eur J Dent. 2013;7(1):41-7.

35. Øgaard B, Larsson E, Henriksson T, Birkhed D, Bishara SE. Effects of combined application of antimicrobial and fluoride varnishes in orthodontic patients. Am J Orthod Dentofacial Orthop. 2001;120(1):28-35.

36. Rohilla M, Pandit IK, Srivastava N. Why the carioprotective potential of luting cements crucial? J Indian Soc Pedod Prev Dent. 2009;27(4):219-23.

37. Rogers S, Chadwick B, Treasure E. Fluoride-containing orthodontic adhesives and decalcification in patients with fixed appliances: a systematic review. Am J Orthod Dentofacial Orthop. 2010;138(4):390.e1-8.

38. Ulkur F, Sungurtekin Ekçi E, Nalbantgil D, Sandalli N. In vitro effects of two topical varnish materials and Er:YAG laser irradiation on enamel demineralization around orthodontic brackets. Sci World J. 2014;2014:490503.

39. Geiger MA, Gorelick L, Gwinnett AJ, Griswold PG. T. The effect of a fluoride program on white spot formation during orthodontic treatment. Am J Orthod Dentofacial Orthop. 1988;93(1):29-37.

40. Heintze SD. A profilaxia individual em pacientes com aparelhos fixos: recomendações para o consultório. Ortodontia. 1996;29(2):4-15.

41. Buonocore MG. A simple method of increasing the adhesion of acrylle filling materials to enamel surfaces. J Dent Res. 1955;34(6):849-53.

42. Bertoz FA, Komatsu J, Okida RC, Mendonça MR. Ionômero de vidro como meio cimentantede bráquetes. Ortodontia. 1991;24(1):41-3.

43. Øgaard B, Rezk-Lega F, Ruben J, Arends J. Cariostatic effect and fluoride release from a visible light-curing adhesive for bonding of orthodontic brackets. Am J Orthod Dentofacial Orthop. 1992;101(4):303-7.

44. Pascotto RC, Navarro MF, Capelozza Filho L, Cury JA. In vivo effect of a resin-modified glass ionomer cement on enamel demineralization around orthodontic brackets. Am J Orthod Dentofacial Orthop. 2004;125(1):36-41.

45. Geiger, AM, Gorelick L, Gwinnett AJ, Benson BJ. Reducing white spot lesions in orthodontic populations with fluoride rinsing. Am J Orthod Dentofacial Orthop. 1992;101(5):403-7.
46. Shannon IL. Prevention of decalcification in orthodontic patients. J Clin Orthod. 1981;15(10):694-705.

47. Sudjalim TR, Woods MG, Manton DJ. Prevention of white spot lesions in orthodontic practice: a contemporary review. Aust Dent J. 2006;51(4):284-9.

48. Vorhies AB, Donly KJ, Staley RN, Wefel JS. Enamel demineralization adjacent to orthodontic brackets bonded with hybrid glass ionomer cements: an in vitro study. Am J Orthod Dentofacial Orthop. 1998;114(6):668-74.

49. Passalini P, Fidalgo TKS, Caldeira EM, Gleiser R, Nojima MCG, Maia LC. Preventive effect of fluoridated orthodontic resins subjected to high cariogenic challenges. Braz Dent J. 2010;21(3):211-5

50. Sonis AL, Snell W. An evaluation of a fluoride-releasing, visible light-activated bonding system for orthodontic bracket placement. Am J Orthod Dentofacial Orthop. 1989;95(4):306-11. 